\title{
CONTROL CURRENT METHOD OF THE CONCENTRATION OF FERROMAGNETIC ELEMENTS IN THE WORKING CHAMBER OF THE TECHNOLOGICAL INDUCTOR OF MAGNETIC FIELD DURING ITS OPERATION
}

Introduction. A rotating magnetic field three-phase inductor designed for the technological processing of various substances is considered. The processing is carried out by ferromagnetic elements in the form of pieces of iron wire moving with a magnetic field. Problem. The control problem of the concentration of ferromagnetic elements in the working chamber of the inductor is solved. This is necessary in order to replenish the chamber in time with elements that wear out but without interrupting the processing. Methodology. The proposed control method consists in observing the current of the inductor stator winding, which is carried out during its operation and does not require intervention in the technological process of processing. Results. The theoretical substantiation of the method is given and a practical calculation evaluation of its adequacy is made on the example of a specific inductor. The theory and practice of the method are based on numerical calculations of the magnetic field, electromagnetic parameters and the angular characteristics of the inductor. Practical value. The practical use of the method is to automatically determine the time of reloading the chamber with ferromagnetic elements that are worn out during the inductor operation. This increases the performance of the inductor and eliminates its downtime. References 10, figures 8.

Key words: magnetic field inductor, working chamber, concentration of ferromagnetic elements, current control, numerical field analysis, electromagnetic parameters, angular characteristics.

Представлений метод визначення концентрації феромагнітних елементів в робочій камері індуктора обертового магнітного поля, призначеного для технологічної обробки різних речовин. Метод полягає в контролі струму обмотки статора індуктора і не вимагає втручання в технологічний процес обробки. Надано теоретичне обгрунтування методу і проведена практична розрахункова оцінка його адекватності на прикладі конкретного індуктора. Теорія $i$ практика методу засновані на чисельних розрахунках магнітного поля, електромагнітних параметрів $і$ кутових характеристик індуктора. Практичне використання методу полягас у визначенні часу додаткового завантажсення камери феромагнітними елементами, які стираються в процесі роботи індуктора. Бібл. 10, рис. 8.

Ключові слова: індуктор магнітного поля, робоча камера, концентрація феромагнітних елементів, контроль струму, чисельно-польовий аналіз, електромагнітні величини, кутові характеристики.

Представлен метод определения концентрации ферромагнитных элементов в рабочей камере индуктора врацающегося магнитного поля, предназначенного для технологической обработки различных веществ. Метод заключается в контроле тока обмотки статора индуктора и не требует вмешательства в технологический процесс обработки. Дано теоретическое обоснование метода и проведена практическая расчетная оценка его адекватности на примере конкретного индуктора. Теория и практика метода основаны на численных расчетах магнитного поля, электромагнитных параметров и угловых характеристик индуктора. Практическое использование метода заключается в определении времени дозагрузки камеры ферромагнитными элементами, которые истираются в процессе работы индуктора. Библ. 10, рис. 8 .

Ключевые слова: индуктор магнитного поля, рабочая камера, концентрации ферромагнитных элементов, токовый контроль, численно-полевой анализ, электромагнитные величины, угловые характеристики.

Introduction. In a number of industries, rotating magnetic field inductors (RMFIs) have become widespread for the processing of various substances. Such inductors are part of various mixers, grinders and separators [1-3].

The stator design of the RMFI is similar to the stator of a three-phase asynchronous motor. Inside it is a cylindrical working chamber through which the processed substance is passed in the axial direction. Ferromagnetic elements (FEs) in the form of pieces of iron wire are used for processing. They are ferromagnetic and move with a rotating field, creating a so-called «eddy layer» in the chamber $[1,3]$. The chamber shell is made of non-magnetic steel and does not interact with the magnetic field.

The magnetic field in the chamber provides not only the movement of the FEs, but also keeps them on the active length of the inductor, which is approximately equal to the axial length of the stator ferromagnetic core.

For the operation of the inductor, a certain amount of FEs is poured into the working chamber, depending on the properties of the processed substance and the intensity of the processing mode. For various reasons, considered in [1], the filling of the chamber with elements decreases with time, and their periodic replenishment is required, which is done through a special pipe without switching off the inductor.

In this case, the inductor under consideration is intended for an apparatus with an «eddy layer» that treats waste water, as described in [1]. In this case, the wear of FEs is predetermined by the friction of their ends against the chamber shell, which even leads to the appearance of through annular grooves in the shell. There are other wear factors, but they are not significant by comparison. The abrasion of the FE ends in the fundamental work [1] was defined by the term «wear of length».

One of the problems of using the described apparatus is the task of when and in what quantity it is necessary to replenish the chamber with a new portion of the FEs.

A known solution is the installation of measuring coils on the bore of the stator core. An EMF is induced in 
them by a rotating magnetic field, which should change with a decrease in the FE concentration and, therefore, a change in the magnetic properties of the medium in the chamber. Thanks to this, the operator or control system receives information that contributes to the solution of the specified task.

However, in practice the described method turns out to be insensitive. And it was not sufficiently substantiated by calculation tests, due to the serious assumptions of the method based on the theory of magnetic circuits used to calculate magnetic fields.

The situation changed with the development of a technique for calculating electromagnetic parameters and characteristics of RMFI based on numerical calculations of magnetic fields, which is presented in the authors' works $[4,5]$. Due to this, it was revealed that it is possible to predict the change in the current of the stator winding of the inductor with a change in the concentration of the FEs in the working chamber. This was confirmed in [6] when studying a relatively small experimental model of the inductor.

The goal of this work is a theoretical substantiation and calculation confirmation of the adequacy of the method for predicting the current concentration of FEs in the working chamber of the RMFI based on monitoring the current of its stator winding. This is carried out on the basis of numerical calculations of the magnetic field and electromagnetic parameters of the inductor.

Object of study. The RMFI electromagnetic system is shown in Fig. 1 by its cross section. The rectangular $(x, y)$ and polar $(r, \alpha)$ coordinate systems used are shown.

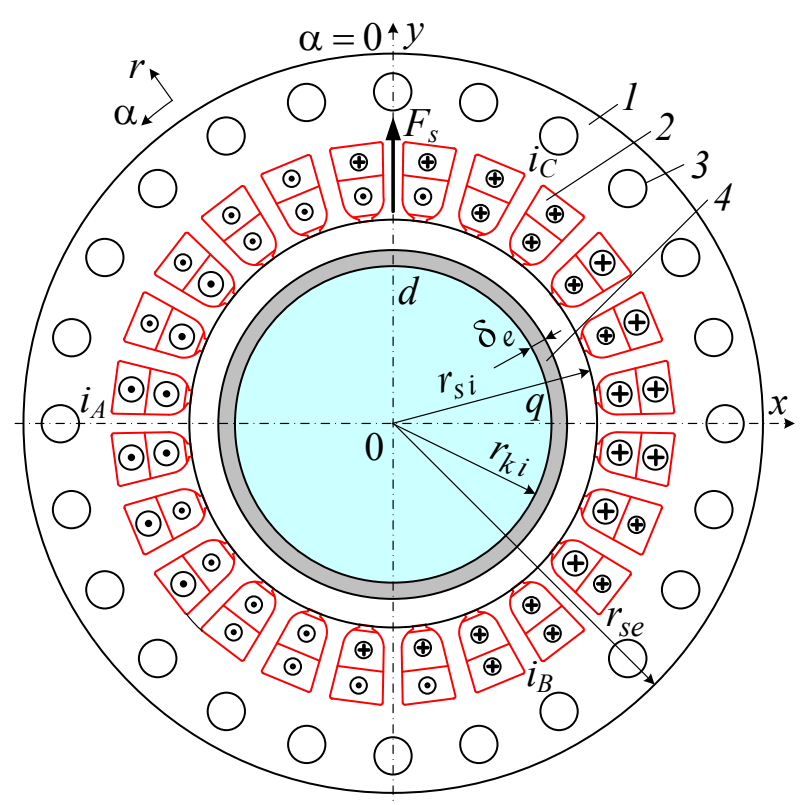

Fig. 1. Electromagnetic system of the RMFI: 1 - laminated stator core; 2 - three-phase winding; 3 - ventilation ducts; 4 - shell of the working chamber with thickness $\delta_{e}=5 \mathrm{~mm}$

The inductor is a three-phase two-pole. For technological reasons, the radius of the inner surface of the chamber $r_{k i}=0.047 \mathrm{~m}$ and the axial length of the stator core $l_{a}=0.25 \mathrm{~m}$ are given. The radii of the core bore $r_{s i}=0.06 \mathrm{~m}$ and its outer surface $r_{s e}=0.109 \mathrm{~m}$ are calculated. The inductor winding is two-layer with a relative shortening of $10 / 12$, the connection diagram is «star», there are 72 turns per phase. The core is made of electrical steel grade 2212 , sheet thickness $0.5 \mathrm{~mm}$, filling factor $K_{F e}=0.95$.

A fragment of an idealized structure with a uniform FE distribution in the working chamber is shown in Fig. 2. The real structure of the elements will be less strict [6], and idealization, as in $[1,4]$, is necessary for an accessible organization of calculations.

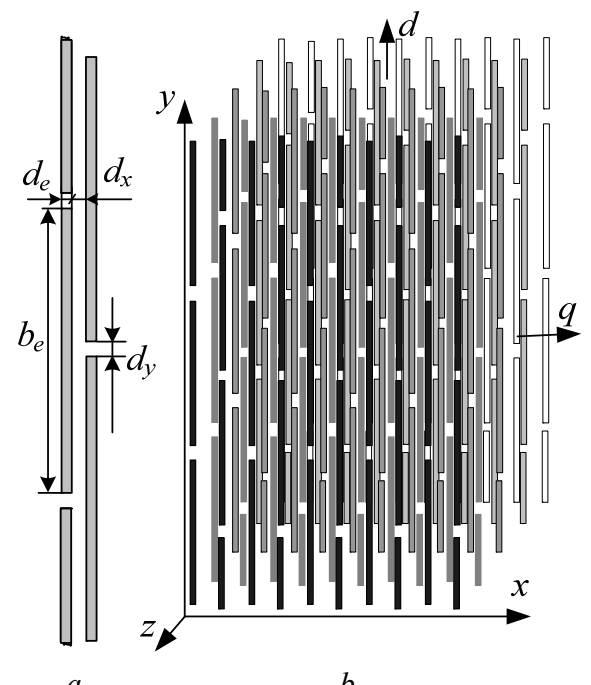

Fig. 2. Fragment of an idealized structure of ferromagnetic elements

FEs are made of steel grade St3. Their dimensions and clearances are taken according to Fig. 2,a: $d_{e}=1 \mathrm{~mm}$; $b_{e}=20 \mathrm{~mm} ; d_{x}=1.5 \mathrm{~mm} ; d_{y}=1 \mathrm{~mm}$. In this case, the filling factor of the chamber with them in the $x y$ plane (Fig. $2, b) K_{F e s}=0.322$. In the $y z$ plane, the same factor is adopted, and then the volumetric filling factor is 0.104 .

In the cross section of the RMFI (see Fig. 1), the magnetic field is considered to be plane-parallel. Therefore, the round sections of the FEs are replaced by square ones with appropriate resizing, as described in [4].

The nominal phase voltage of the stator winding $U_{s N}$ is $100 \mathrm{~V}$ at frequency of $f_{s}=50 \mathrm{~Hz}$. In ideal idle mode, i.e. in the absence of ferromagnetic elements in the working chamber, the average value of the magnetic flux density $B_{a v}$ is set and is $0.12 \mathrm{~T}$, which corresponds to practically used inductors.

Basics of numerical field calculations. The source of the rotating magnetic field in the inductor is a threephase symmetric system of currents of phase stator windings (see Fig. 1):

$$
\begin{gathered}
i_{A}=I_{m} \cos \left(\omega_{s} t+\beta\right) ; \\
i_{B}=I_{m} \cos \left(\omega_{s} t-2 \pi / 3+\beta\right) ; \\
i_{C}=I_{m} \cos \left(\omega_{s} t+2 \pi / 3+\beta\right),
\end{gathered}
$$

where $t$ is time; $I_{m}=\sqrt{2} I_{s}$ is the amplitude of the phase currents; $I_{s}$ is their effective value; $\omega_{s}=2 \pi f_{s}$ is the angular frequency; $\beta$ is the initial phase of the currents, which sets the angular displacement of the direction of the MMF of the stator winding $F_{s}$ from the $y$ axis which is necessary for a particular calculation mode. 
Figure 1 shows the directions of currents in the phase windings in the ideal idle mode, at which $\beta=0$, and therefore the MMF vector $F_{s}$ is directed along the $y$-axis.

The magnetic field of the inductor in its central cross section is described by the well-known 2D differential equation:

$$
\operatorname{rot}\left[\mu_{a}^{-1} \operatorname{rot}\left(\vec{k} A_{z}\right)\right]=\vec{k} J_{z},
$$

where $\vec{k}$ is the ort along the axial $z$-axis; $A_{z}, J_{z}$ are the components of the magnetic vector potential (MVP) and current density; $\mu_{a}$ is the absolute magnetic permeability.

The propagation of the magnetic field is limited by the outer surface of the inductor core, where the Dirichlet boundary condition is set: $A_{z}=0$.

The tool of the presented research is the numerical calculations of the magnetic field by the Finite Element Method using the FEMM code [7] with its control by the created Lua script, as in [8].

Moreover, in contrast to $[4,5]$, here the calculation of the field itself is carried out with direct account of a discrete medium with FEs in the working chamber, instead of replacing it with an equivalent continuous medium. This eliminates the assumptions and additional errors associated with such a replacement.

The assumption of the description of the magnetic field by equation (2) is justified by calculations in [9] and experimentally confirmed in [10], and in this inductor the ratio of the axial length and diameter of the core bore $l_{a} / d_{s i}$ is 2.3 and 3.2 times larger, respectively, which reduces possible calculation error.

When calculating the field, the magnetic permeability $\mu_{a}$ in the laminated steel core and in the FEs is taken into account by a well-known method, taking into account the magnetization curves of steels. The filling factors of the core $K_{F e}$ and the working chamber $K_{F e s}$ along the $z$ axis are set as initial parameters for the FEMM code, and are taken into account by its software.

The phase relations of the electromagnetic quantities of the inductor and the essence of the angle $\beta$ are considered in detail in $[4,5]$. It is shown that when the inductor is operating under load, the angle $\beta$ is in the range of $0-90^{\circ}$, and at extreme values, the electromagnetic torque (EMT) is absent. This corresponds to the mode of the operating idle [5], which differs from the ideal idle by the presence of ferromagnetic elements in the chamber.

Figures 3, 4 show the results of calculating the magnetic field in the mode of operating idle and in the nominal load mode at the indicated values of the stator current $I_{s}$ and angle $\beta$. In Fig. 5 the magnetic field in the working chamber is shown on an enlarged scale for the nominal load mode.

In fact, the EMT in the considered inductor is reactive, and its principle of operation corresponds to a synchronous reluctance motor, which is substantiated in $[4,5]$. Therefore, in the working chamber, the longitudinal axis $d$ is assigned in the direction of the FEs orientation, and the transverse axis $q$ is perpendicular to it (see Fig. 1). In the steady-state operating mode, the axes rotate together with these elements and the field with a frequency $n_{s}$.

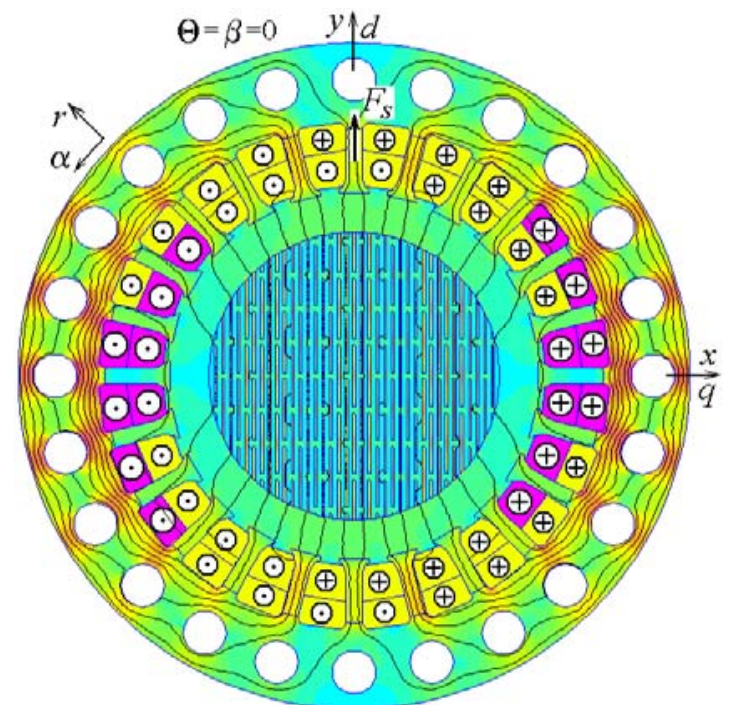

Fig. 3. Magnetic field in the inductor in the mode of the working idle at $I_{s}=35 \mathrm{~A}$ and $\beta=0$

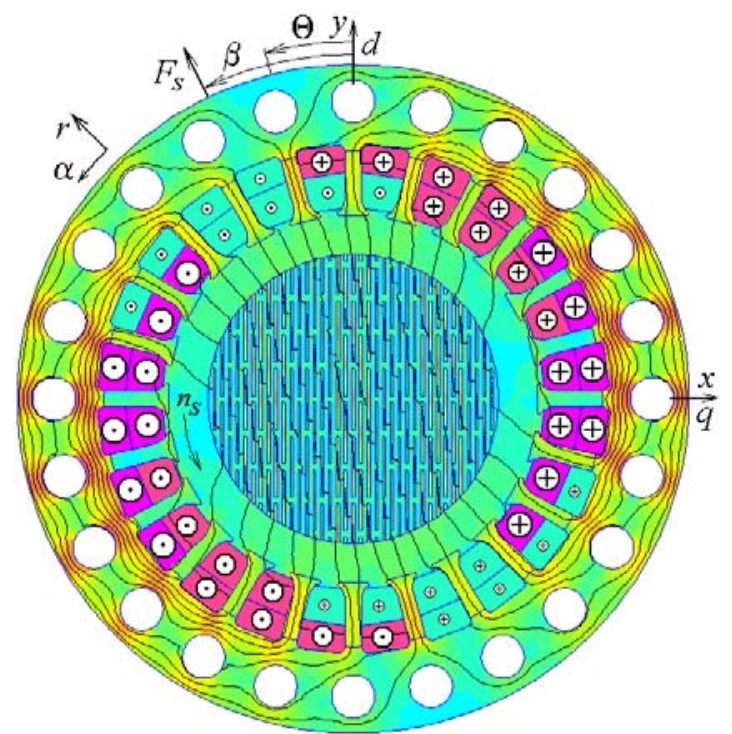

Fig. 4. Magnetic field in the inductor in the nominal load mode at $I_{s}=36 \mathrm{~A}$ and $\beta=26.7^{\circ}$

In Fig. 4, 5 the deviation of the field lines from the longitudinal axes of the Fes is shown. This is a condition for the occurrence of the EMT which acts on the entire mass of FEs and sets them in motion. The nature of this moment corresponds to Maxwell's theory of the magnetic tension tensor, which is explained in [5].

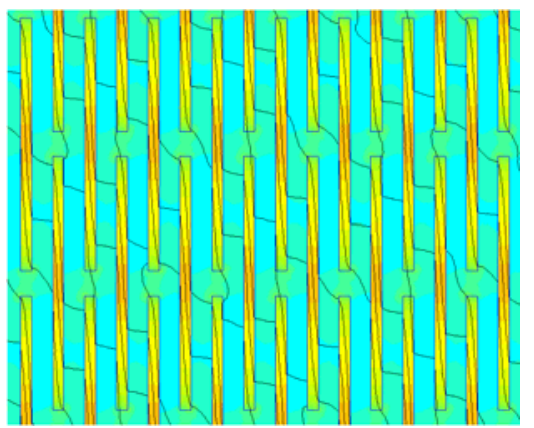

Fig. 5. Fragment of the picture of the magnetic field in the working chamber of the inductor at nominal load 
Electromagnetic quantities in the RMFI are determined on the basis of the method described in [5], and for this work the following provisions are in demand from it.

According to the results of calculating the magnetic field using the FEMM code [7], the EMT is obtained through the Maxwell tensor of magnetic tension:

$$
M_{e m}=\frac{l_{a}}{\mu_{0}\left(r_{s i}-r_{k i}\right)} \int_{S_{\delta}} B_{r} B_{\alpha} r d S,
$$

where $B_{r}$ and $B_{\alpha}$ are the radial and angular components of the magnetic flux density; $S_{\delta}$ is the cross-sectional area of the gap, limited by circles with radii $r_{k i}$ and $r_{s i}$ on the sides of the chamber and stator; $\mu_{0}$ is the magnetic constant.

According to the MVP distribution, the time function of the first harmonic of the magnetic flux linkage of the phase stator winding is formed:

$$
\Psi_{a}=\Psi_{m} \cos \left(\omega_{s} t+\gamma_{\psi a}\right) \text {. }
$$

In accordance with the law of electromagnetic induction, from (4) the EMF of this winding is obtained:

$$
e_{a}=\omega \Psi_{m} \cos \left(\omega_{s} t+\gamma_{\psi a}-\pi / 2\right),
$$

whence its effective value and initial phase:

$$
E_{a}=\sqrt{2} \pi f_{s} \Psi_{m} ; \gamma_{E a}=\gamma_{\psi a}-\pi / 2 .
$$

The balance of voltages in the phase stator winding is represented by the complex equation [4]:

$$
\underline{U}_{s}=-\underline{E}_{a}+j X_{v} \underline{I}_{s}+\left(R_{s}+R_{\text {mag }}\right) \underline{I}_{s},
$$

where on the basis of (1) and (6) the complexes of its current $\underline{I}_{S}=I_{s} e^{j \beta}$ and EMF $\underline{E}_{a}=E_{a} e^{j \gamma_{E a}}$ appear.

The formula (7) includes the active resistance $R_{s}$ of the stator winding and the reactance of its frontal dissipation $X_{v}$, which are calculated as in induction motors: $R_{s}=0.31 \Omega$ and $X_{v}=0.39 \Omega$. The active resistance $R_{\text {mag }}$ reflects the power of the magnetic losses and is determined during the iterative calculation of the magnetic field.

By voltage (7), its effective value $U_{s}$ and phase shift relative to current $\varphi_{s}=\gamma_{U_{s}}-\beta$ are found in exponential form $\underline{U}_{S}=U_{s} e^{j \gamma_{U s}}$.

After calculating the magnetic field and electromagnetic parameters in the considered load mode of the RMFI at $U_{s}=U_{s N}$ and indicated in Fig. 4 values $I_{s}$ and $\beta$ we obtain: $R_{\text {mag }}=0.01207 \Omega ; \Psi_{m}=0.376 \mathrm{~Wb}$; $\gamma_{\Psi a}=15.08^{\circ} ; E_{a}=83.6 \mathrm{~V} ; \varphi_{s}=73.5^{\circ}$.

In $[4,5]$ it was revealed that when setting $\beta=0$, the value $\gamma_{\Psi a}$ turned out to be the same, and this corresponded to the mode of operating idle, since the EMT was equal to zero, too. The angle of rotation of the magnetic flux linkage vector $\Psi_{a}$ from the idle mode to the load mode, according to the theory of synchronous machines, is the load angle $\Theta$. Therefore, the angle $\gamma_{\Psi a}$ in (4) is the load angle of the RMFI, i.e. $\Theta=\gamma_{\Psi a}$. The relationship of the angles $\beta$ and $\Theta$ with each other, as well as with the load torque and other electromagnetic parameters of the inductor are presented in detail in $[4,5]$.

Determination of the current factor of filling the working chamber with ferromagnetic elements.

As an indicator of the filling of the working chamber, it is proposed to use the current of the stator winding, and more specifically, the change in the current of the phase winding. To identify such a possibility, a series of corresponding calculations of the magnetic field and electromagnetic parameters of the inductor was performed according to the above method with formulae (3) $-(7)$.

The process of wear of ferromagnetic elements in this work is simulated in the simplest version by changing their length, which is already denoted as $b_{e}$, and the number of FEs in the chamber is considered unchanged.

Previously, the results of calculations have already been presented for a basic value of the length $b_{e}$ equal to $20 \mathrm{~mm}$ and selected values of the angle $\beta$ with the corresponding current $I_{s}$. Now, to this value of $b_{e}$, we add four more values of the FE length as it decreases with a step of $2 \mathrm{~mm}$. And we carry out the calculations for a number of $\beta$ values in order to obtain the angular characteristics of the inductor. In this case, naturally, the gaps $d_{y}$ between the elements increase accordingly, and the diameter of the elements $d_{e}$ and the gaps $d_{x}$ between them remain the same.

Figure 6 shows the models of the modernized filling of the working chamber (symmetric quarters of the cross section) with the corresponding patterns of magnetic fields. For the basic filling option, a similar full image is shown in Fig. 4 and in the form of a fragment - in Fig. 5.

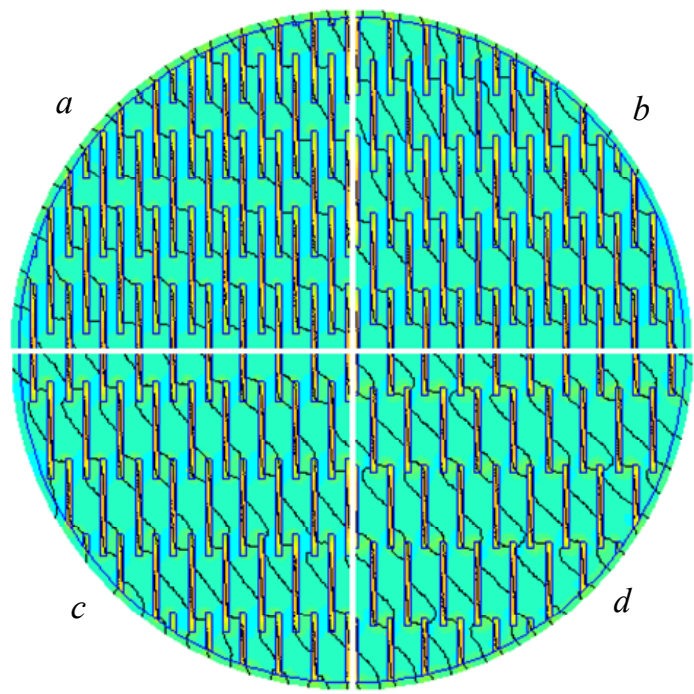

Fig. 6. Working chamber filling options with a change in the length of the ferromagnetic elements: $a-b_{e}=18 \mathrm{~mm} ; b-b_{e}=16 \mathrm{~mm} ; c-b_{e}=14 \mathrm{~mm} ; d-b_{e}=12 \mathrm{~mm}$

With the accepted modifications of the FEs, the filling factor of the working chamber in the cross section $K_{F e s}$ changes, but the filling factor in the longitudinal section $K_{F e s z}$ remains, as in the basic version $-K_{F e s b}$. Then the volumetric filling factor of the chamber is defined as $K_{F e v}=K_{F e s} \cdot K_{F e s b}$.

The initial parameters of the five calculation options, namely: $b_{e}, K_{F e s}$ and $K_{F e v}$, are given in Table 1 .

At the given voltage of the stator winding for five specific options for filling the working chamber, the families of the two angular characteristics of the inductor required in this case were calculated, as in [5]: $I_{s}(\Theta)$ и $M_{e m}(\Theta)$ - they are shown in Fig. 7. 
Table 1

Dependencies of the parameters of the inductor on the length of ferromagnetic elements

\begin{tabular}{|c|c|c|c|c|c|c|}
\hline \multirow{2}{*}{ Option } & \multirow{2}{*}{$\begin{array}{c}b_{e}, \\
\mathrm{~mm}\end{array}$} & \multirow{2}{*}{$K_{F e s}$} & $K_{F e v}$ & \multicolumn{2}{|c|}{$I_{s}, \mathrm{~A}$} & \multirow{2}{*}{$E_{a}, \mathrm{~V}$} \\
\cline { 5 - 6 } & & & & $M_{\text {emo }}=0$ & $M_{\text {emN }}$ & \\
\hline 1 & 20 & 0.322 & 0.104 & 35.0 & 36.0 & 83.6 \\
\hline 2 & 18 & 0.290 & 0.094 & 38.4 & 39.4 & 82.6 \\
\hline 3 & 16 & 0.256 & 0.082 & 41.1 & 42.1 & 81.1 \\
\hline 4 & 14 & 0.226 & 0.073 & 43.5 & 44.1 & 80.1 \\
\hline 5 & 12 & 0.193 & 0.062 & 45.8 & 46.5 & 79.1 \\
\hline
\end{tabular}

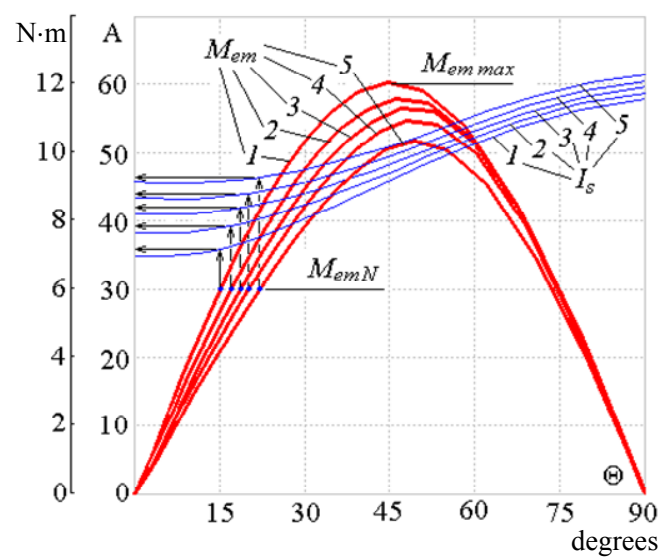

Fig. 7. Plotting on angular characteristics of the current $I_{s}$ and EMT $M_{e m}$ to obtain the dependence $I_{s}\left(b_{e}\right)$ at nominal EMT $M_{e m N}$

The technique for calculating the characteristics was as follows.

The generalized initial phase of currents $\beta$ included in (1), which depends on the load level of the inductor $[4,5]$, is taken as a variable value.

At an unchanged voltage $U_{s}$, for each value of the angle $\beta$, the inverse problem of calculating the magnetic field is solved resulting in the required value of the current $I_{s}$. This solution, as shown in [5], is achieved by the method of successive approximations with the solution at each iteration of the direct problem - by calculating the magnetic field for a given value of the angle $\beta$ and the selected value of the current $I_{s}$, and, therefore, the known distribution of currents in the windings according to (1).

At a specific value of the angle $\beta$, the initial approximation of the current $I_{s}$ is set and the magnetic field is calculated, and as a result, using (7), the value of the voltage $U_{s}$ is obtained. It is compared with the set value $U_{s N}$ and the current value $I_{s}$ is corrected depending on the result. The iterations continue until the coincidence of $U_{s}$ and $U_{s N}$ with the desired accuracy, which is described in [5].

The characteristics of the inductor were calculated in the range of angle $\beta$ from 0 to $90^{\circ}$ by passing it with a step of $5^{\circ}$. For each $\beta$ value, the corresponding values of the current $I_{s}$, EMT $M_{e m}$, EMF $E_{a}$ and the load angle $\Theta$ were determined, which is further taken as an argument for the characteristics $I_{s}(\Theta)$ and $M_{e m}(\Theta)$.

To reveal the dependence $I_{s}\left(b_{e}\right)$ at the nominal electromagnetic torque $M_{e m N}$, the constructions shown in Fig. 7 are carried out. The $M_{e m N}$ value was $6 \mathrm{~N} \cdot \mathrm{m}$, and it was assumed to be equal to half the maximum torque $M_{\text {emmax }}$ at the base length of the ferromagnetic elements $b_{e}=20 \mathrm{~mm}$.

The results obtained - the values of the current $I_{s}$ at $M_{e m N}$ and at the accepted values of $b_{e}$ are summarized in Table 1. Here are also given the values of the current $I_{s}$ at a zero value of the torque $M_{\text {emo }}$, which corresponds to the previously described mode of the operating idle at $\beta=0$.

Available now according to the Table 1 two dependencies $I_{s}\left(b_{e}\right)$ at $M_{e m N}$ and $M_{e m o}$ are shown graphically in Fig. 8. Obviously, all other permissible modes of operation will be within the range between the resulting two graphs.

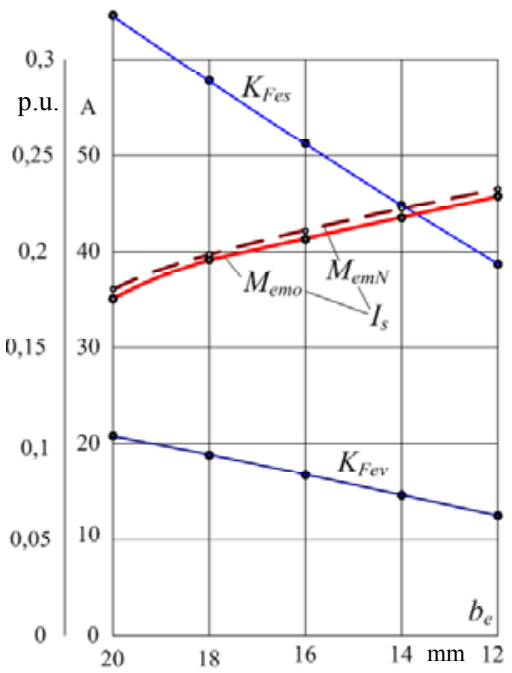

Fig. 8. Dependencies of the filling factors of the working chamber $K_{F e s}$ and $K_{F e v}$ and the stator winding current $I_{s}$ on the FE length $b_{e}$

Here, according to Table 1, the graphs of the dependencies of the planar $K_{F e s}$ and volumetric $K_{F e v}$ filling factors of the working chamber are also plotted.

Now according to the Table 1 and Fig. 8, it can be seen that in the considered range of variation of the length of the ferromagnetic elements, the stator winding current will automatically change to a rather noticeable value of about $10 \mathrm{~A}$. It can be easy recorded continuously using an ammeter or a special current sensor.

Thus, the operator controlling the inductor or the automatic control system can determine the current value of the filling factor of the working chamber with ferromagnetic elements and, therefore, the moment in time when it is necessary to replenish it with new elements.

Also, in the Table 1 the column of the EMF of phase stator winding $E_{a}(6)$ is added. In the considered range of variation in the length of ferromagnetic elements, this EMF changed by $5.4 \%$. The EMF will also change to the same extent of any measuring coils, which are used to indicate the filling of the working chamber of the inductor with ferromagnetic elements.

But the phase current at the same time changed to $29.2 \%$ - therefore, this is namely a more visual and sensitive indicator of the concentration of ferromagnetic elements in the working chamber, that is, an indicator of the volumetric filling factor of the working chamber $K_{\mathrm{Fev}}$ which in Table 1 changed by $40 \%$. 
The results obtained are qualitatively confirmed by experimental data on a simplified inductor model [6]. In the conditions of the studies carried out there, it was revealed that a decrease in the volumetric filling factor of the chamber from 0.037 to 0 led to an increase in the stator current by $13.4 \%$. The smaller change in comparison with the RMFI considered in the paper is explained by the significantly lower values of the filling factor $k_{\mathrm{FeV}}$.

\section{Conclusions.}

1. The current method developed on the basis of numerical field calculations of magnetic fields makes it possible to relate the concentration of ferromagnetic elements in the working chamber of the rotating magnetic field inductor and the current in its winding.

2. Practical calculations on a specific sample of the inductor showed that the wear of the length of the ferromagnetic elements due to their abrasion by $40 \%$ leads to an automatic increase in the phase current by $29.2 \%$. At the same time, the applied alternative control method by means of measuring coils requires a more complex inductor design and gives a decrease in EMF only by $5.4 \%$, that is, it has a much lower sensitivity.

3. Observation of the current of the inductor winding makes it possible to control the filling of its working chamber with ferromagnetic elements without interrupting the process of operation of the RMFI. This makes it possible to timely replenish the chamber with such elements and, thereby, maintain the technological processing of various substances passed through this chamber at a given level.

4. Despite the fact that the studies were carried out for a specific version of FE wear (length wear), the proposed method of current control of the filling of the working chamber with them can be applied for other wear options. For example, when the FEs are thinned, when the decreases in the length and thickness of the FEs are combined, or simply when the FEs are removed from the working chamber.

\section{REFERENCES}

1. Logvinenko D.D., Sheljakov O.P. Intensifikacija tehnologicheskih processov $v$ apparatah $s$ vihrevym sloem [Intensification of technological processes in apparatus with a vortex layer]. Kiev, Tehnika Publ., 1976. 144 p. (Rus).

2. Belounis A., Mehasni R., Ouil M., Feliachi M., El-Hadi Latreche M. Design with optimization of a magnetic separator for turbulent flowing liquid purifying applications. IEEE Transactions on Magnetics, 2015, vol. 51, no. 8, pp. 1-8. doi: 10.1109/TMAG.2015.2424401.

3. Gerasimov M.D., Loktionov I.O. Dual-use technological solutions. Application prospects. Vektor GeoNauk, 2019, vol. 2, no. 1, pp. 19-26. (Rus). doi: 10.24411/2619-0761-2019-10003.

4. Milykh V.I., Shilkova L.V. Numerical-field analysis of the characteristics of a three-phase magnetic field inductor for the treatment of various substances with current stabilization. Electrical engineering \& electromechanics, 2019, no. 6, pp. 2128. (Rus). doi: 10.20998/2074-272X.2019.6.03.

5. Milykh V.I., Shilkova L.V. Characteristics of a cylindrical inductor of a rotating magnetic field for technological purposes when it is powered from the mains at a given voltage. Electrical engineering \& electromechanics, 2020, no.2, pp. 13-19. (Rus). doi: 10.20998/2074-272X.2020.2.02.

6. Milykh V.I., Shilkova L.V. Experimental research of the three-phase physical model of the magnetic field inductor in the working mode when processing bulk material. Bulletin of NTU "KhPI». Series: "Electric machines and electromechanical energy conversion», 2020, no.3(1357), pp. 3-7. (Ukr). doi: 10.20998/2409-9295.2020.3.01.

7. Finite Element Method Magnetics: OldVersions. FEMM 4.2 11 oct2010 Self-Installing Executable. Available at: http://www.femm.info/wiki/OldVersions (accessed 15.06.2017).

8. Milykh V.I. The system of automated formation of electrical machines computational models for the FEMM software environment. Technical Electrodynamics, 2018, no.4, pp. 74-78. (Ukr). doi: 10.15407/techned2018.04.074.

9. Milykh V.I., Shilkova L.V. Revuzhenko S.A. Numerical analysis of the magnetic field of a cylindrical three-phase magnetic separator inductor. Bulletin of NTU «KhPI». Series: «Electric machines and electromechanical energy conversion», 2017, no. 1(1223), pp. 76-82. (Ukr).

10. Milykh V.I., Shilkova L.V. Numerical-experimental analysis of the magnetic field of a magnetic separator inductor on the basis of an asynchronous motor. Bulletin of NTU «KhPI». Series: «Electric machines and electromechanical energy conversion», 2018, no. 5(1281), pp. 104-109. (Ukr).

Received 16.07.2020

V.I. Milykh ${ }^{1}$, Doctor of Technical Science, Professor,

L.V. Shilkova ${ }^{1}$, Postgraduate Student,

${ }^{1}$ National Technical University «Kharkiv Polytechnic Institute»,

2, Kyrpychova Str., Kharkiv, 61002, Ukraine,

e-mail: mvikemkpi@gmail.com, larisa_lv@ukr.net

How to cite this article:

Milykh V.I., Shilkova L.V. Control current method of the concentration of ferromagnetic elements in the working chamber of the technological inductor of magnetic field during its operation. Electrical engineering \& electromechanics, 2020, no. 5, pp. 12-17. doi: 10.20998/2074-272X.2020.5.02. 